\title{
Numerical Strategies for the System of First Order IVPs Using Block Hybrid Extended Trapezoidal Multistep Method of Second Kind for Stiff ODEs
}

\author{
Yohanna Sani Awari \\ Department of Mathematical Sciences, Taraba State University, Jalingo, Nigeria
}

Email address:

awari04c@yahoo.com

To cite this article:

Yohanna Sani Awari. Numerical Strategies for the System of First Order IVPs Using Block Hybrid Extended Trapezoidal Multistep Method of Second Kind for Stiff ODEs. Science Journal of Applied Mathematics and Statistics. Vol. 5, No. 5, 2017, pp. 181-187.

doi: 10.11648/j.sjams.20170505.13

Received: September 18, 2017; Accepted: October 8, 2017; Published: November 8, 2017

\begin{abstract}
A Modified Three Step Block Hybrid Extended Trapezoidal Multistep Method of Second Kind (BHETR 2 s) with two off-grid points, one at interpolation and another at collocation point yielding uniform order six $(6,6,6,6,6)^{\mathrm{T}}$ for the Numerical Integration of initial value problems of stiff Ordinary Differential Equations was developed. The main method and additional equations were obtained from the same continuous formulation through interpolation and collocation procedures. The stability properties of the method was discussed and from the stability region obtained, the method is suitable for the solution Stiff Ordinary Differential Equations. Three numerical examples were considered to illustrate the efficiency and accuracy.
\end{abstract}

Keywords: Collocation, A-Stability, Hybrid Method, Initial Value Problem, Stiff Differential Equations

\section{Introduction}

Consider the stiff initial value problem in the form:

$$
y^{\prime}(x)=f(x, y), y(a)=y_{0}
$$

on the finite interval $I=\left[x_{0}, x_{N}\right]$, where

$$
y:\left[x_{0}, x_{N}\right] \rightarrow R^{m} \quad \text { and } \quad f:\left[x_{0}, x_{N}\right] \times R^{m} \rightarrow R^{m} \quad \text { are }
$$

continuous. By considering the partition

$$
I_{j}=a+j h, j=0, \ldots, N-1, h=\frac{b-a}{N-1}
$$

one can consider the $k$-step LMM

$$
\sum_{j=0}^{k} \alpha_{j} y_{n+j}=h\left[\sum_{j=0}^{k} \beta_{j}(x) f_{n+j}\right]
$$

to approximate the solution of problem (1) over the considered partition. As usual, $y_{n+j}$ and $f_{n+j}$ denote the approximations to $y\left(x_{n+j}\right)$ and $f\left(x_{n+j}, y\left(x_{n+j}\right)\right)$ respectively.
The initial value problem of stiff differential equations occurs in almost every field of science [see 3, 8, 10, 12 and 15], particularly, in the fields of:

A) Chemical Reactions: A famous chemical reaction is the Oregenator reaction between $\mathrm{HBrO}_{2}, \mathrm{Br}^{-}$, and $\mathrm{Ce}$ (IV) described by Field and Noyes in 1984.

B) Reaction-diffusion systems: Problems in which the diffusion is modeled via the Laplace operator may become stiff as they are discretize in space by finite differences or finite elements, well-known example of such systems which appear so often in mathematical biology.

Several further occurrences of stiffness can be found in electrical circuits, mechanics, meteorology, oceanography and vibrations.

Definition 1: If the solution of the system contains components which change at significantly different rates for given changes in the independent variable, then system is said to be stiff $[8,15]$.

Stiff differential equations are characterized as those whose exact solution has a term of the form $e^{-\lambda t}$, where $\lambda$ is a large positive constant. The key features of stiff equations are that the derivative terms may increase rapidly as $t$ 
increases [12].

In the last three decades numerous works have been focusing on the development of more advanced and efficient methods for stiff problems $[8,12]$. The situation becomes more complicated when stiffness coupled with nonlinearity. Carroll presents an exponential fitted scheme for solving stiff systems of initial value problems [15]. The numerical solution of linear and nonlinear system of stiff system can be found in [3, 11 and 12].

Block methods were introduced to both improve the stability of methods and provide the $k-1$ starting values to $k-$ step LMM. They can be seen as a set of linear multistep methods simultaneously applied to (1) and then combined to yield better approximations (Ajie, et al., 2014).

\section{Formulation of the Method}

A more elegant and computationally attractive procedurewas proposed in this paper, which leads to a class of stable general linear methods for stiff systems of initial value problems. Although the method was formulated in terms of multistep collocation methods, yet they preserve many of the Runge-Kutta properties, such as being self-starting and of permitting easy change of step length during implementation.

The exact solution $\mathrm{y}(\mathrm{x})$ is approximated by seeking the continuous metho $\mathrm{d} \bar{y}(x)$ of the form

$$
y(x)=\sum_{j=0}^{k-1} \phi_{j}(x) y_{n+j}+\phi_{q}(x) y_{n+q}+h\left[\sum_{j=k-2}^{2} \psi_{j}(x) f_{n+j}+\psi_{q}(x) f_{n+q}\right]
$$

here, $\phi_{j}(x), \psi_{j}(x)$ for $j=0(1) 2$ and $\psi_{q}(x), \phi_{q}(x)$ are coefficients of the method which are to be determined. $q=\frac{a}{b}$, a rational number in the form $\frac{2 r+1}{2}, r=1,2$ where

$$
\begin{aligned}
& \phi_{0}(x)=1-\frac{1225}{303} \frac{\xi}{h}+\frac{8035}{1212} \frac{\xi^{2}}{h^{2}}-\frac{3419}{606} \frac{\xi^{3}}{h^{3}}+\frac{3187}{1212} \frac{\xi^{4}}{h^{4}}-\frac{193}{303} \frac{\xi^{5}}{h^{5}}+\frac{19}{303} \frac{\xi^{6}}{h^{6}} \\
& \phi_{1}(x)=-\frac{22016}{101} \frac{\xi^{3}}{h^{3}}+\frac{17268}{101} \frac{\xi^{2}}{h^{2}}-\frac{3876}{101} \frac{\xi^{5}}{h^{5}}+\frac{13329}{101} \frac{\xi^{4}}{h^{4}}-\frac{5040}{101} \frac{\xi}{h}+\frac{436}{101} \frac{\xi^{6}}{h^{6}} \\
& \phi_{2}(x)=-\frac{4017}{101} \frac{\xi^{5}}{h^{5}}+\frac{60687}{404} \frac{\xi^{2}}{h^{2}}-\frac{40631}{202} \frac{\xi^{3}}{h^{3}}+\frac{475}{101} \frac{\xi^{6}}{h^{6}}-\frac{4365}{101} \frac{\xi}{h}+\frac{52203}{404} \frac{\xi^{4}}{h^{4}} \\
& \phi_{\frac{3}{2}}(x)=\frac{29440}{303} \frac{\xi}{h}-\frac{99328}{303} \frac{\xi^{2}}{h^{2}}+\frac{23872}{303} \frac{\xi^{5}}{h^{5}}-\frac{2752}{303} \frac{\xi^{6}}{h^{6}}+\frac{128704}{303} \frac{\xi^{3}}{h^{3}}-\frac{79936}{303} \frac{\xi^{4}}{h^{4}} \\
& \psi_{1}(x)=\frac{11947}{303} \frac{\xi^{4}}{h^{3}}-\frac{6953}{101} \frac{\xi^{3}}{h^{2}}+\frac{17704}{303} \frac{\xi^{2}}{h}-\frac{1940}{101} \xi+\frac{364}{303} \frac{\xi^{6}}{h^{5}}-\frac{1112}{101} \frac{\xi^{5}}{h^{4}} \\
& \psi_{\frac{3}{2}}(x)=\frac{704}{303} \frac{\xi^{2}}{h}-\frac{64}{101} \xi+\frac{704}{303} \frac{\xi^{4}}{h^{3}}+\frac{32}{303} \frac{\xi^{6}}{h^{5}}-\frac{336}{101} \frac{\xi^{3}}{h^{2}}-\frac{8584}{101} \frac{\xi^{3}}{h^{2}}+\frac{1155}{101} \xi-\frac{142}{101} \frac{\xi^{6}}{h^{5}}+\frac{1166}{101} \frac{\xi^{5}}{h^{4}}
\end{aligned}
$$

Evaluating the continuous formulation in (3) yields the $B H E T R_{2} s$ associated with the continuous scheme and converting it into $A, B, U$ and $V$ of the General Linear Method (12) as:

$$
\left[\begin{array}{cccccc|cccc}
0 & 0 & 0 & 0 & 0 & 0 & 0 & 0 & 0 & 1 \\
-\frac{101}{5040} & -\frac{388}{1008} & 0 & \frac{231}{1008} & -\frac{4}{315} & 0 & -\frac{97}{112} & \frac{5888}{3024} & 0 & -\frac{35}{432} \\
0 & -\frac{159}{112} & -\frac{303}{56} & -\frac{387}{244} & \frac{3}{56} & 0 & \frac{4023}{448} & 0 & -\frac{891}{112} & -\frac{11}{448} \\
0 & -\frac{113}{633} & 0 & \frac{530}{1477} & -\frac{256}{4431} & \frac{101}{13293} & 0 & \frac{68864}{39879} & -\frac{152}{211} & -\frac{257}{39879} \\
0 & -\frac{75}{404} & 0 & \frac{675}{808} & \frac{15}{101} & 0 & -\frac{675}{1616} & \frac{225}{101} & -\frac{325}{404} & -\frac{9}{1616} \\
0 & \frac{366}{101} & 0 & -\frac{738}{101} & \frac{192}{101} & 0 & \frac{2187}{101} & -\frac{3584}{101} & \frac{1485}{101} & \frac{13}{101} \\
\hline 0 & \frac{366}{101} & 0 & -\frac{738}{101} & \frac{192}{101} & 0 & \frac{2187}{101} & -\frac{3584}{101} & \frac{1485}{101} & \frac{13}{101} \\
0 & -\frac{113}{633} & 0 & \frac{530}{1477} & -\frac{256}{4431} & \frac{101}{13293} & 0 & \frac{68864}{39879} & -\frac{152}{211} & -\frac{257}{39879} \\
0 & -\frac{159}{112} & -\frac{303}{56} & -\frac{387}{244} & \frac{3}{56} & 0 & \frac{4023}{448} & 0 & -\frac{891}{112} & -\frac{11}{448} \\
-\frac{101}{5040} & -\frac{388}{1008} & 0 & \frac{231}{1008} & -\frac{4}{315} & 0 & -\frac{97}{112} & \frac{5888}{3024} & 0 & -\frac{35}{432}
\end{array}\right]
$$




\section{Order, Convergence and Absolute Stability Region}

\subsection{Order of BHETR $\mathrm{S}_{2}$}

Definition 2

A linear multistep method (3) is consistent if it has order $p \geq 1$.

According to [9], a LMM is said to be of order $p$ if $c_{0}=c_{1}=c_{2}=\ldots c_{p}=0, c_{p+1} \neq 0$, this approach can be extended to determine the order of the entire block method which can be expressed as:

$$
\sum_{i=0}^{k} \alpha_{i j} y_{n+j}=h \sum_{i=0}^{k} \beta_{i j} f_{n+j}
$$

where, $j=0,1, \ldots, k$ is a positive integer, equation (5) can be expanded to give the following system of equation

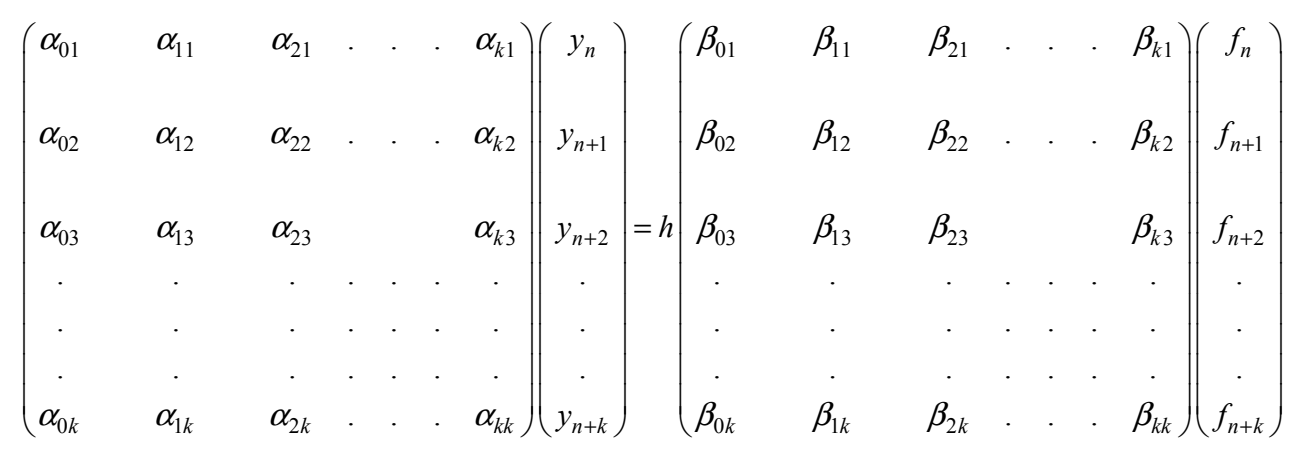

The expression (6) is equivalent to (5) where,

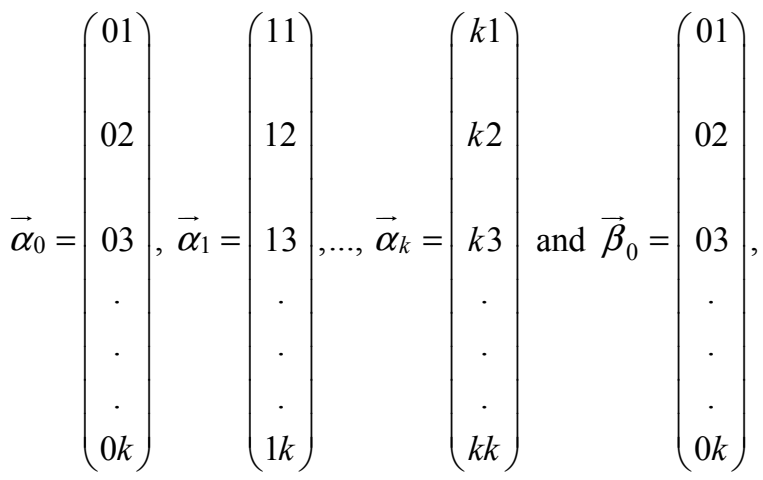

$$
\vec{\beta}_{1}=\left(\begin{array}{c}
11 \\
12 \\
13 \\
\cdot \\
\cdot \\
\cdot \\
1 k
\end{array}\right), \ldots, \vec{\beta}_{k}=\left(\begin{array}{c}
k 1 \\
k 2 \\
k 3 \\
\cdot \\
\cdot \\
k k
\end{array}\right)
$$

Adopting the order procedure used in the single case for the block method and recall that

$$
L_{h} y(x)=\sum_{i=0}^{k}\left[\bar{\alpha}_{j} y(x+j h)-h \bar{\beta}_{j} f(x+j h, y(x+j h))\right]
$$

where, $y(x)$ is the exact solution satisfying (1). Carrying out
Taylor series expansion on (8) about $x$ yields the equation

$$
\begin{aligned}
& L_{h} y(x)= \vec{c}_{0} y(x)+\vec{c}_{1} h y^{\prime}(x)+\vec{c}_{2} h^{2} y^{\prime \prime}(x)+\ldots+\vec{c}_{q} h^{q} y^{(q)}(x)(9) \\
& c_{02} \\
& \vec{c}_{0}=\left(\begin{array}{c}
c_{01} \\
c_{03} \\
\cdot \\
\cdot \\
\cdot \\
c_{0 p}
\end{array}\right), \vec{c}_{12}=\left(\begin{array}{c}
c_{11} \\
c_{13} \\
\cdot \\
\cdot \\
\cdot \\
c_{1 p}
\end{array}\right), \ldots, \vec{c}_{p}=\left(\begin{array}{c}
c_{p 2} \\
c_{p 3} \\
\cdot \\
\cdot \\
\cdot \\
c_{p p}
\end{array}\right)
\end{aligned}
$$

From (4), the coefficients of (7) are obtained as

$$
\begin{aligned}
\vec{\alpha}_{0} & =\left(\begin{array}{cccccc}
-\frac{13}{101} & \frac{9}{1616} & \frac{257}{303} & -\frac{11}{2424} & \frac{1225}{303} & 0
\end{array}\right)^{T} \\
\vec{\alpha}_{1} & =\left(\begin{array}{lllllll}
-\frac{1485}{101} & \frac{325}{404} & \frac{9576}{101} & -\frac{297}{202} & \frac{5040}{101} & 0
\end{array}\right)^{T} \\
\vec{\alpha}_{\frac{3}{2}} & =\left(\begin{array}{lllllll}
\frac{3584}{101} & \frac{225}{101} & -\frac{68864}{303} & -\frac{56}{303} & -\frac{29440}{303} & 0
\end{array}\right)^{T} \\
\vec{\alpha}_{2} & =\left(\begin{array}{ccccccc}
-\frac{2187}{101} & \frac{675}{1616} & \frac{13293}{101} & \frac{1341}{303} & \frac{4365}{101} & 0
\end{array}\right)^{T} \\
\vec{\alpha}_{3}=\left(\begin{array}{ccccccc}
1 & 0 & 0 & 0 & 0 & 0
\end{array}\right)^{T} &
\end{aligned}
$$




$$
\vec{\alpha} \frac{5}{2}=\left(\begin{array}{llllll}
0 & 1 & 0 & 0 & 0 & 0
\end{array}\right)^{T}
$$

and

$$
\begin{aligned}
& \vec{\beta}_{0}=\left(\begin{array}{llllll}
0 & 0 & 0 & 0 & -1 & 0
\end{array}\right)^{T} \\
& \vec{\beta}_{1}=\left(\begin{array}{llllll}
\frac{366}{101} & -\frac{150}{808} & -\frac{2373}{101} & \frac{106}{404} & -1 & 0
\end{array}\right)^{T} \\
& \vec{\beta}_{\frac{3}{2}}=\left(\begin{array}{llllll}
0 & 0 & 0 & 1 & 0 & 0
\end{array}\right)^{T} \\
& \vec{\beta}_{2}=\left(\begin{array}{llllll}
-\frac{738}{101} & \frac{675}{808} & \frac{4770}{101} & \frac{129}{404} & \frac{1155}{101} & 0
\end{array}\right)^{T} \\
& \vec{\beta}_{3}=\left(\begin{array}{llllll}
0 & 0 & 1 & 0 & 0 & 0
\end{array}\right)^{T} \\
& \vec{\beta}_{\frac{5}{2}}=\left(\begin{array}{llllll}
\frac{192}{101} & \frac{120}{808} & -\frac{768}{101} & -\frac{4}{404} & -\frac{64}{101} & 0
\end{array}\right)^{T}
\end{aligned}
$$

Applying (8)-(10), yields a uniformly sixth order for the BHETR $_{2} S$, which is presented in table (1).

Definition 3A-Stability[4]

A numerical method (4) is said to be A-stable if its region of absolute stability contains, the whole of the left-hand half plane $\operatorname{Reh} \lambda<0$

Definition 4

The method presented in (4) is said to be of order $p$ if $c_{0}=c_{1}=c_{2}=\ldots=c_{p}$ and $c_{p+1} \neq 0, c_{p+1}$ is called the error constant and the local truncation error given by

$$
T_{n}=\vec{c}_{p+1} h^{p+1} y^{(p+1)}\left(x_{n}\right)
$$

\subsection{Absolute Stability Region}

To determined the absolute stability region of the block method, they are reformulated into General Linear Methods of [2] where they used as partition $(s+r)(s+r)$ matrix containing $A, B, U$ and $V$ expressed as (4) in the form

$$
\left[\frac{Y}{y^{i-1}}\right]=\left[\begin{array}{ll}
A & U \\
B & V
\end{array}\right]\left[\frac{h f(Y)}{y^{i}}\right], i=1,2, \ldots, N
$$

Where the matrices $A, B, U$ and $V$ are substituted into a stability matrix

$$
M(z)=V+z B(I-z A)^{-1} U
$$

Which is in-turn substituted into a stability function

$$
\rho(\lambda, z)=\operatorname{det}(\lambda I-M z)
$$

The values of $A, U, B, V$ in (7) are substituted in (5) to obtained the stability matrix. Plotting the stability matrix in MATLAB codeto obtained the region of absolute stability of the block hybrid method as shown in Figure 1.

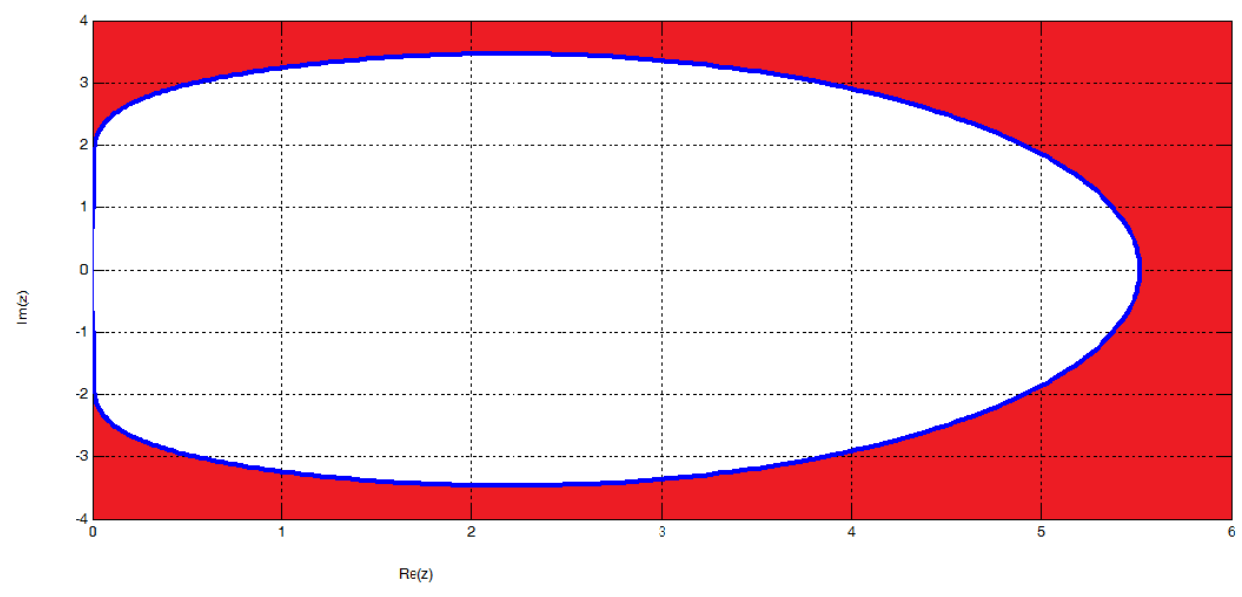

Figure 1. Region of absolute stability of the block hybrid method.

Note:

a. Figure 1 presents the region of absolute stability of the block hybrid method and is shown to be the entire shaded portion including the left hand half complex plane (in agreement with definition 3)

a. Analysis from the graph (Figure 1) suggests that the block hybrid method proposed in this paper would be suitable to solve stiff ordinary differential equations.

\subsection{Convergence}

Following [5], the BHETR ${ }_{2} \mathrm{~s}$ (4) can be represented by a matrix finite difference equation in the form:

$$
A^{(0)} y_{m+1}=\sum_{i=1}^{k} A^{(i)} y_{m+1}+h \sum_{i=0}^{k} B^{(i)} f_{m-1}
$$

where 


$$
\begin{aligned}
& A^{(0)}=\left[\begin{array}{lllll}
1 & 0 & 0 & 0 & 0 \\
0 & 1 & 0 & 0 & 0 \\
0 & 0 & 1 & 0 & 0 \\
0 & 0 & 0 & 1 & 0 \\
0 & 0 & 0 & 0 & 1
\end{array}\right], A^{(1)}=\left[\begin{array}{lllll}
0 & 0 & 0 & 0 & 1 \\
0 & 0 & 0 & 0 & 1 \\
0 & 0 & 0 & 0 & 1 \\
0 & 0 & 0 & 0 & 1 \\
0 & 0 & 0 & 0 & 1
\end{array}\right], \\
& B_{1}=\left[\begin{array}{ccccc}
\frac{673}{360} & -\frac{104}{45} & \frac{211}{120} & -\frac{32}{45} & \frac{43}{360} \\
\frac{1323}{640} & -\frac{77}{40} & \frac{1053}{640} & -\frac{27}{40} & \frac{73}{640} \\
\frac{92}{45} & -\frac{224}{135} & \frac{29}{15} & -\frac{32}{45} & \frac{16}{135} \\
\frac{2375}{1152} & -\frac{125}{72} & \frac{875}{384} & -\frac{35}{72} & \frac{125}{1152} \\
\frac{81}{40} & -\frac{8}{5} & \frac{81}{40} & 0 & \frac{11}{40}
\end{array}\right] \text { and } \\
& B_{0}=\left[\begin{array}{ccccc}
0 & 0 & 0 & 0 & \frac{11}{40} \\
0 & 0 & 0 & 0 & \frac{35}{128} \\
0 & 0 & 0 & 0 & \frac{37}{135} \\
0 & 0 & 0 & 0 & \frac{35}{128} \\
0 & 0 & 0 & 0 & \frac{11}{40}
\end{array}\right]
\end{aligned}
$$

Definition 5: A block method is zero stable provided the roots $\lambda_{i j}=1,2, \ldots, k$ of the first characteristic polynomial $\rho(\lambda)$ specified as

$$
\rho(\lambda)=\operatorname{det}\left[\sum_{i=0}^{k} A^{(i)} \lambda^{k-i}\right]=0
$$

satisfies $\left|\lambda_{j}\right| \leq 1$, the multiplicity must not exceed two, [6]

Following (16), we have that

$$
\begin{gathered}
\rho(\lambda)=\operatorname{det}\left[\lambda\left[\begin{array}{lllll}
1 & 0 & 0 & 0 & 0 \\
0 & 1 & 0 & 0 & 0 \\
0 & 0 & 1 & 0 & 0 \\
0 & 0 & 0 & 1 & 0 \\
0 & 0 & 0 & 0 & 1
\end{array}\right]-\left[\begin{array}{lllll}
0 & 0 & 0 & 0 & 1 \\
0 & 0 & 0 & 0 & 1 \\
0 & 0 & 0 & 0 & 1 \\
0 & 0 & 0 & 0 & 1 \\
0 & 0 & 0 & 0 & 1
\end{array}\right]\right] \\
\rho(\lambda)=\operatorname{det}\left[\left[\begin{array}{lllll}
\lambda & 0 & 0 & 0 & -1 \\
0 & \lambda & 0 & 0 & -1 \\
0 & 0 & \lambda & 0 & -1 \\
0 & 0 & 0 & \lambda & -1 \\
0 & 0 & 0 & 0 & \lambda-1
\end{array}\right]\right] \\
\rho(\lambda)=\lambda^{4}(\lambda-1)=0
\end{gathered}
$$

Thus, $\lambda_{1}=1, \lambda_{2}=\lambda_{3}=\lambda_{4}=\lambda_{5}=0$. By definition 5 , the BHETR $_{2} \mathrm{~s}$ method is zero stable.

\section{Implementation Strategies}

In this section, we have tested the performance of our three-step block method on three (3) numerical Initial Value Problems of first order ODEs. For each example; we find the absolute errors of the approximate solution.

Example 4.1: We consider the Initial Value Problem with step-size $h=0.1$

$$
\frac{d y}{d x}-x y=0, y(0)=1
$$

Analytical Solution of the given problem is $y(x)=e^{\frac{x^{2}}{2}}$

Table 1. Maximum Errors for Example 4.1.

\begin{tabular}{lll}
\hline $\mathbf{X}$ & Maximum Error in[13] & Maximum Error in the New Block Hybrid Method \\
\hline 0.1 & $5.29 \mathrm{E}-007$ & $3.165 \mathrm{E}-009$ \\
0.2 & $1.77 \mathrm{E}-007$ & $3.172 \mathrm{E}-009$ \\
0.3 & $8.99 \mathrm{E}-007$ & $3.439 \mathrm{E}-009$ \\
0.4 & $3.09 \mathrm{E}-007$ & $1.625 \mathrm{E}-008$ \\
0.5 & $1.91 \mathrm{E}-006$ & $1.683 \mathrm{E}-008$ \\
0.6 & $4.48 \mathrm{E}-006$ & $1.843 \mathrm{E}-008$ \\
0.7 & $1.02 \mathrm{E}-005$ & $5.456 \mathrm{E}-008$ \\
0.8 & $7.74 \mathrm{E}-005$ & $5.833 \mathrm{E}-008$ \\
0.9 & $1.44 \mathrm{E}-005$ & $6.522 \mathrm{E}-008$ \\
1.0 & $2.93 \mathrm{E}-005$ & $1.668 \mathrm{E}-007$ \\
\hline
\end{tabular}

Example 4.2: The SIR model is an epidemiological model that computes the theoretical numbers of people infected with a contagious illness in a closed population over time. The name of this class of models derives from the fact that they involves coupled equations relating the number of susceptible people $S(t)$, number of people infected $I(t)$ and the number of people who have recovered $R(t)$. This is a good and simple model for many infectious diseases including measles, mumps and rubella [13-15]. The SIR 
model is described by the three coupled equations.

$$
\begin{aligned}
& \frac{d s}{d t}=\mu(1-S)-\beta I S \\
& \frac{d I}{d t}=-\mu I-\gamma I+\beta I S \\
& \frac{d s}{d t}=-\mu R+\gamma I
\end{aligned}
$$

where $\mu, \gamma$ and $\beta$ are positive parameters.

Define $y$ to be $y=S+I+R$
Adding all these equations give

$$
y^{\prime}=\mu(1-y)
$$

Taking $\mu=0.5$ and attaching an initial condition $y(0)=0.5$ (for a particular closed population), we obtain

$$
y^{\prime}(t)=0.5(1-y), y(0)=0.5
$$

Whose analytical solution is $y(t)=1-0.5 e^{-0.5 t}$

Table 2. Maximum Errors for Example 4.2.

\begin{tabular}{lllll}
\hline $\mathbf{X}$ & Analytical Solution & $\begin{array}{l}\text { Numerical Solutionforthe New } \\
\text { Block Hybrid Method }\end{array}$ & Maximum Error in [18] & $\begin{array}{l}\text { Maximum Error in the New Block } \\
\text { Hybrid Method }\end{array}$ \\
\hline 0.1 & 0.524385287749643 & 0.524385287750215 & $5.574430 \mathrm{E}-012$ & $5.72 \mathrm{E}-013$ \\
0.2 & 0.547581290982020 & 0.547581290982560 & $3.946177 \mathrm{E}-012$ & $5.40 \mathrm{E}-013$ \\
0.3 & 0.569646011787471 & 0.569646011787959 & $8.183232 \mathrm{E}-012$ & $4.88 \mathrm{E}-013$ \\
0.4 & 0.590634623461009 & 0.590634623461945 & $3.436118 \mathrm{E}-011$ & $9.36 \mathrm{E}-013$ \\
0.5 & 0.610599608464298 & 0.610599608465186 & $1.929743 \mathrm{E}-010$ & $8.88 \mathrm{E}-013$ \\
0.6 & 0.629590889659141 & 0.629590889659952 & $1.879040 \mathrm{E}-010$ & $8.11 \mathrm{E}-013$ \\
0.7 & 0.647655955140644 & 0.647655955141798 & $1.776835 \mathrm{E}-010$ & $1.154 \mathrm{E}-012$ \\
0.8 & 0.664839976982180 & 0.664839976983277 & $1.724676 \mathrm{E}-010$ & $1.097 \mathrm{E}-012$ \\
0.9 & 0.681185924189114 & 0.681185924190118 & $3.005775 \mathrm{E}-010$ & $1.004 \mathrm{E}-012$ \\
1.0 & 0.696734670143684 & 0.696734670144944 & $1.260 \mathrm{E}-012$ \\
\hline
\end{tabular}

Example 4.3: Consider the test problem $\frac{d y}{d x}-\lambda y=0, y(0)=1$, with solution $y=e^{\lambda x}$ is solved with $h=0.01$ and $\lambda=-5$.

Table 3. Maximum Errors for Example 4.3.

\begin{tabular}{lllll}
\hline X & Analytical Solution & $\begin{array}{l}\text { Numerical Solutionforthe New } \\
\text { Block Hybrid Method }\end{array}$ & $\begin{array}{l}\text { Maximum Error in } \\
\text { [1]0rder 7 }\end{array}$ & $\begin{array}{l}\text { Maximum Error in the New Block } \\
\text { Hybrid Method0rder6 }\end{array}$ \\
\hline 0.02 & 0.904837418035960 & 0.904837418034642 & $8.57 \mathrm{E}-012$ & $1.318 \mathrm{E}-012$ \\
0.04 & 0.818730753077982 & 0.818730753075408 & $7.79 \mathrm{E}-012$ & $2.574 \mathrm{E}-012$ \\
0.06 & 0.740818220681718 & 0.740818220679243 & $7.00 \mathrm{E}-012$ & $2.475 \mathrm{E}-012$ \\
0.08 & 0.670320046035639 & 0.670320046032424 & $1.33 \mathrm{E}-011$ & $3.215 \mathrm{E}-012$ \\
0.10 & 0.606530659712633 & 0.606530659708700 & $1.18 \mathrm{E}-011$ & $3.933 \mathrm{E}-012$ \\
1.12 & 0.548811636094026 & 0.548811636090359 & $1.07 \mathrm{E}-011$ & $3.667 \mathrm{E}-012$ \\
\hline
\end{tabular}

Table 4. Order and Error Constants of the Method.

\begin{tabular}{lll}
\hline Method & Uraer $\boldsymbol{p}$ & Error Cons tan ts \\
\hline & 6 & $\frac{71}{56560}$ \\
4 & 6 & $-\frac{15}{361984}$ \\
& 6 & $-\frac{243}{28280}$ \\
& 6 & $\frac{29}{1357440}$ \\
& 6 & $\frac{107}{33936}$ \\
\hline
\end{tabular}

\section{Conclusions}

We have developed a modified three-step block hybrid extended trapezoidal multistep method of second kind with two off-grid points, yielding uniform order six (table 4) for the numerical integration of initial value problems of stiff ordinary differential equations. The new block methods are self- starting and all the discrete schemes used were obtained from the single continuous formulation and its derivative which are of uniform order of accuracy. Implementation of our method in block form tends to speed up computational process. Results obtained from our method shows significant improvement when compared with results of existing 
authors. Our sixth order block hybrid method performs better than the seventh order method of Ajie et al., (see table 3).

\section{Reference}

[1] Ajie I. J., Ikhile M. N. O and Onumanyi P. A Family of OneBlock Implicit Multistep Backward Euler Type Methods American Journal of Computational and Applied Mathematics 2014, 4(2): 51-59.

[2] Butcher, J. C. and Burrage, K. (1980). Non-Linear Stability of a General Class of Differential Equation Methods. BIT, 20: 185-203.

[3] Carroll, J. "A Metrically Exponentially Fitted Scheme for the Numerical Solution of Stiff Initial Value Problems," Computers \& Mathematics with Applications, Vol. 26, 1993, pp. 57-64. doi:10.1016/0898-1221(93)90034-S

[4] Dahlquist, G. (1963). A Special Stability Problem for Linear Multistep Methods. BIT., 3,27-43.

[5] Fatunla, S. O. (1991). Block methods for second order IVP's. International Journal of Computer Mathematics, 41, 55-63.

[6] Fatunla, S. O. (1994). Higher Order Parallel Methods for Second Order ODE's. Scientific Computing. Proceeding of fifth International Conference on Scientific Computing.

[7] Gragg, W. B. and Stetter, H. J. Generalized Multistep Predictor-Corrector Methods. Journal of Association of Computing Machines, Vol. 11, No. 2, 1964, pp. 188-209.

[8] Harier, E. and Wanner, G. "Solving Ordinary Differential Equations II, Stiff and Differential-Algebraic Problems," Springer-Verlag, New York, 1996. doi:10.1007/978-3-64205221-7.

[9] Henrici, P. (1962). Discrete Variable Methods in ODE's (1st Edition). Wiley and Sons Ltd., New York. pp. 285.
[10] Hojjati, G., Rahimi, M. Y. and Hosseini, S. M. "An Adaptive Method for Numerical Solution of Stiff System of Ordinary Differential Equations," Mathematics and Computers in Simulation, Vol. 66, No. 1, 2004, pp. 33-41. doi:10.1016/j.matcom.2004.02.019.

[11] Hsiao, C. H. and Wang, W. J. "Haar Wavelet Approach to Non-Linear Stiff Systems," Mathematics and Computer in Simulation, Vol. 57, No. 6, 2001, pp. 347-353. doi:10.1016/S0378-4754(01)00275-0.

[12] Hsiao, C. H. "Haar Wavelet Approach to Linear Stiff Systems," Mathematics and Computer in Simulation, Vol. 64, No. 1, 2004, pp. 561-567. doi:10.1016/j.matcom.2003.11.011.

[13] James, A. A., Adesanya, A. O. and Joshua, S. Continuous block method for the solution of second order initial value problems of ordinary differential equations, Int. J. of pure and Appl. Math. 88(2013), 405-416.

[14] James, A. A., Adesanya, A. O., Sunday, J., Yakubu, D. G. Half-Step Continuous Block Method for the Solutions of Modeled Problems of Ordinary Differential Equations, American Journal of Computational Mathematics, 2013, 3, 261-269.

[15] Lopidus, L. and Schiesser, W. E. "Numerical Methods for Differential Systems," Academic Press, New York, 1976.

[16] Mickens, R. E. Non-Standard Finite Difference Models of Differential Equations. World Scientific, Singapore, 1994.

[17] Rosser, J. B. A Runge-kutta Method for All Seasons, SIAM Review, Vol. 9, No. 3, 1967, pp. 417-452.

[18] Sunday, J., Odekunleans, M. R. and Adesanya, A. O. Order Six Block Integrator for the Solution of First Order Ordinary Differential Equations. IJMS, Vol. 3, No. 1 2013, pp. 87-96. 